\title{
Co-Designing Flavor-Based Memory Cues with Older Adults
}

\author{
Tom Gayler \\ School of Computing and \\ Communications \\ Lancaster University \\ Lancaster, UK \\ t.gayler@lancaster.ac.uk
}

\author{
Corina Sas \\ School of Computing and \\ Communications \\ Lancaster University \\ Lancaster, UK \\ c.sas@lancaster.ac.uk
}

\author{
Vaiva Kalnikaite \\ Dovetailed \\ Cambridge, UK \\ vaiva@dovetailed.io
}

\begin{abstract}
This initial study explores the design of flavor-based cues with older adults for their self-defining memories. It proposes using food to leverage the connections between odor and memory to develop new multisensory memory cues. Working with 4 older adults, we identified 6 self-defining autobiographical memories for each participant, 3 related to food, 3 unrelated to food. Flavorbased cues were then created for each memory through a codesign process. Findings indicate the dominance of relationship themes in the identified self-defining memories and that flavorbased cues related mostly to multiple ingredient dishes. We discuss how these findings can support further research and design into flavor-based memory cues through 3D food printing.
\end{abstract}

\section{CCS CONCEPTS}

-Human-centered computing Human computer interaction (HCI) •Human-centered computing Interaction design Interaction design process and methods $\sim$ Participatory design

\section{KEYWORDS}

Food; Co-design; Self-defining memories; Older adults; Memory cues; Multisensory design; Odor; Flavor.

\section{ACM Reference format:}

Tom Gayler, Corina Sas and Vaiva Kalnikaite. 2020. Co-Designing FlavorBased Memory Cues with Older Adults. In Proceedings of ICMI'20 Companion. ACM, New York, NY, USA, 5 pages. https://doi.org/10.1145/3395035.3425644

\section{Introduction}

A wealth of research emphasizes the value of smell for prompting the recall of personal memories [17,37], in particular the role of odor cues for autobiographical memory and their neural correlates $[2,5,14,17,22]$. However, the prevalence of visual, aural Permission to make digital or hard copies of all or part of this work for personal or classroom use is granted without fee provided that copies are not made or distributed for profit or commercial advantage and that copies bear this notice and the full citation on the first page. Copyrights for components of this work owned by others than the author(s) must be honored. Abstracting with credit is permitted. To copy otherwise, or republish, to post on servers or to redistribute to lists, requires prior specific permission and/or a fee. Request permissions from Permissions@acm.org.

ICMI '20 Companion, October 25-29, 2020, Virtual event, Netherlands (-) 2020 Copyright is held by the owner/author(s). Publication rights licensed to ACM. ACM ISBN 978-1-4503-8002-7/20/10...\$15.00

https://doi.org/10.1145/3395035.3425644 and haptic modalities, has left the sense of smell less explored for the design of interactive systems. Given the challenges of providing odor stimuli within computing systems such as delivery, repeatability of stimuli and a lack of effective design tools [3,25], this work explores the potential of 3D printed food to provide odor based memory cues. We report on preliminary findings of a study with 4 participants co-designing personalized flavor-based memory cues for their self-defining, i.e., emotional autobiographical memories. The study engages older adults (over60) who are at higher risk of mild cognitive impairments [34]. The aim of this research is to explore flavor-based cues as part of memory aid systems and food as a novel modality with unique qualities that may support HCI research on memory technologies [20]. This study sets out to answer two questions:

- What self-defining memories are selected to be cued with a flavor-based cue?

- What flavor-based cues are designed for self-defining memories which include food and do not include food?

\section{Related Work}

\subsection{Human-Food Interaction in HCI}

Food as a resource for design has received growing HCI interest $[1,4,11]$ given its value for supporting emotional communication [12]. By focusing on the act of eating food; the mouth and the body can themselves become sites for interactive experience, moving towards more integrated conceptions of how bodies and technology can interact [11]. One of the drivers of the growth of food in $\mathrm{HCI}$ is the development of new technologies. For instance, $3 \mathrm{D}$ food printing has been used to support emotional experience [10] as well as communication between lovers [12] and the potential for flavor-based memory cues has been suggested. Such cues could support narrative and temporal experiences with food [11] curated around personally meaningful memories [10,12].

\subsection{HCI Research on Memory Cues}

A memory cue is something that serves the function of triggering memory recall [20]. Cues have been designed across a range of application domains in HCI, one of which is supporting those with memory impairments associated with aging [15] in part due to impairments in autobiographical memories having knock-on negative effects on an individual's sense of self [28]. Self-defining memories are a type of autobiographical memory that features 
rich emotional qualities and support a person's sense of self [28] and are thus important to preserve. HCI work exploring the potential benefit of such memories and their cues [29] extends Hoven \& Eggen's work on autobiographical memory and their design recommendations for segmented memory systems [19]. The specific modalities of memory cues have been of great interest in HCI $[13,21,26,36]$, including how different sensory pathways may offer benefits, not only in terms of accuracy of memory retrieval, but also the emotional aspects that make a memory feel more 'real' [17]. While visual and aural cues have been much explored [23,30-32], the use of sense of smell in $\mathrm{HCI}$ as a powerful route to memory cueing $[7,25]$ has received less attention, in part due to challenges of implementing odorant-based systems that require sourcing of specialist volatile fragrances [25]. Food, however, is a much more common and accessible material with which to craft odor-based memory cues and via tools such as $3 \mathrm{D}$ food printing, can be integrated within interactive systems [7]. Food also supports the recommendations for memory cues [19], including by being physical $[21,36]$, both in the sense that it is material stuff, but also in the way that it is experienced by the body. It is highly diverse as a resource for design that can support adaptive [21] applications, sensitive to cultural and personal perspectives [26]. Through the use of $3 \mathrm{D}$ food printing it is also possible to envisage usage and experiences both user-initiated [21] and unsolicited $[13,19]$.

\subsection{Food, Odor and Memory Relationship}

Food's connection with memory comes from the flavor experience, which is a compound multisensory experience that is mostly derived from our sense of smell [35]. Therefore food experience is closely linked to the experience of odors that have been shown to be a strong cue for memory $[2,5,14,17,22]$. This is due in part to the neural physiology resulting in direct connections between the olfactory bulb and the parts of the brain related to memory $[2,18]$. However, as of yet there is limited evidence into the function of food as a memory cue. Previous work has explored claims that odors represent the 'best' memory cues [16,17] based on comparisons with verbal, visual, and tactile cues [16]. Odor cues were found to be equivalent to other modalities in terms of memory accuracy but more emotional, in particular, countering anecdotal perspectives on the emotionality of tactile cues [16]. In work specifically targeting autobiographical memory, odors were again found to be more emotional and evocative than visual, verbal or auditory cues but no more specific or vivid [17]. Within memory research in $\mathrm{HCI}$ there is increased interest in multisensory cueing, leveraging the capabilities of different modalities to construct more powerful and efficient cues $[23,26]$. Age has been seen to impact on the function of odor-evoked memories, since older adults who were found to have a higher number of autobiographical memories that could be triggered by odors than younger people [38]. This indicates that older adults are prime candidates for interventions with odor-based memory aids, however declining taste and odor sensitivity associated with aging also needs to be considered [6].

\section{Method}

The aim of the study is to explore how to co-design food-based memory cues with older adults. Participants were 4 older adults (aged 60+, 3 females, 1 male) with no taste or memory impairments recruited through convenience sampling.

The study involved two parts. Part 1 focused on sensitizing participants to identify food-based experiences by completing a bespoke visual probe kit to describe and reflect on food-based experiences. The visual probe kit was inspired by previous work [12], and consisted of several parts such as exercises in sensory deprivation, body mapping and flavor description, each intended to explore different aspects of food experience. The kit also invited participants to connect their memories to food-based experiences by selecting specific memories and writing recipes that can best describe them. It was completed over two weeks in participants' homes to allow them to engage with food experiences in their familiar context where most such experiences are usually situated. The probe kit required completion of paper-based documents and the capture of digital images to support participants during the co-design phase.

Part 2 consisted of individual workshops for co-designing personalized flavor-based memory cues for 6 self-defining memories that we elicited: 3 memories included food (FM), for example, a wedding breakfast, and 3 memories which did not include food (NFM), for instance starting at university. Participants first freely recalled each of the 6 memories in as much detail as possible and were prompted further by three questions to capture associated feelings, social context, and event's place and time. Before the co-design of cues for each memory, participants were given samples of 3D printed food similar to the method described in [10], one for each of the 5 basic tastes (sweet, bitter, salty, sour and umami) in order to familiarize participants with them as benchmarks in the co-design process. These small samples were solutions of sugar, coffee, salt, lemon juice and miso paste to represent each basic taste respectively.

For the co-design of cues for the 3 food-related memories, participants were asked to identify and describe the foodstuff involved in these memories using the flavor design sheet. The sheet included space for noting ingredients, flavor descriptors and cooking processes as text, as well as Likert scales to record the taste profile relative to the 5 basic tastes, the texture, and how lingering the flavor should be. When co-designing the cues for non-food related memories, participants had to first describe associations between the memory and foods before they could provide details on the chosen cue. Prompts were given for associations between the memory and food, via connections to related people, feelings, places and events. Possibilities were generated between researcher and participant, with the latter making the decision on the final cue for each of the 3 non-food related memories. The study concluded with semi-structured interviews to reflect on the co-design of flavor-based memory cues for self-defining memories. The interviews and the design process were audio recorded and fully transcribed and preliminary findings from the thematic analysis described below. 


\section{Findings}

\subsection{Food and Non-food Memories}

To understand the characteristics of the memories chosen by participants we classified them according to categories from previous work on older adults' self-defining memories [28,34] which consist of those focused on life-threatening events, relationships, achievement/mastery, redemption, large scale events, guilt/shame, and drug and alcohol. Findings indicate that from the 24 identified self-defining memories, the most frequent theme was relationships ( $75 \%$ of all memories, referenced in $\mathrm{n}=18$ memories, 9 FM, 9 NFM), achievements (29.2\%, $\mathrm{n}=7$, 4 FM, 3 NFM) and life threatening events $(8.3 \%, \mathrm{n}=2,2 \mathrm{NFM}), 3$ memories were classified with more than one theme. We found that both FM and NFM were dominated by relationship themes, often associated with social and recreational experiences. This focus within FM arose from activities such as visiting restaurants for special occasions (P1, Honeymoon Lobster thermidor), holidays abroad with loved ones (P1, Spit-roast chicken in Naples) or having foodbased celebrations ( $\mathrm{P} 2$, mackerel $\mathrm{BBQ}$ at anniversary party). The dominance of relationship type amongst NFM reflects previous work on self-defining memories with older adults [28].

Memories were overwhelmingly emotionally positive $(87.5 \%, \mathrm{n}=$ 21, 12 FM, 9 NFM), and even more so for food related ones, which were all positive compared to $75 \%$ of NFM. While most memories related to experiences in small groups (2-3 collocated people) $(58 \%$, $\mathrm{n}=14,7$ FM, 7 NFM), large group ones were less frequent $(29 \%, \mathrm{n}=7$, 4 FM, 3 NFM) and alone experiences the least (13\%, n=3, 1 FM, 2 NFM). Such sociality aspects did not differ significantly between food and non-food related memories. There were a similar number of food related and non-food related memories for both one-off events $(87.5 \%, \mathrm{n}=21,10 \mathrm{FM}, 11 \mathrm{NFM})$ and for repeated ones $(12.5 \%$, $\mathrm{n}=3$, 2 FM, 1 NFM). Self-defining memories by their nature are often singular events but the inclusion of the repeated events can be attributed to how repeated interactions with food, such as the crisp sandwich at Grandma's house (P4) or salty crisps eaten at the seaside (P2), can elevate repeated events to meaningful memories [13]. Surprisingly, memories were more weighted towards those from adulthood (71\%, 8 from middle age, 9 from older adulthood), with only $29 \%$ of memories coming from before, or during the 'reminiscence bump', as suggested by previous findings on odor-cued memories [22]. In addition, our findings also confirm those indicating greater prevalence of autobiographic memories related to odors amongst older adults [38], probably due to their greater cumulated experience. This outcome is important as it indicates the potential of food to address the self-defining memories' bias towards earlier life [28] and to support these memories along the entire life span.

\subsection{Flavor-based Memory Cues}

Participants co-designed a flavor-based cue for each of their 6 selfdefining memories (3 FM and 3 NFM). For each cue participants described the relative levels of the 5 basic tastes. Findings indicate that umami was the highest or joint highest for $13 / 24$ of the designed cues, salty for 9 , sweet 8 , with sour and bitter for 1 , each.
The preference for umami and salty type flavors for most of the positive experiences contrasts with previous work which indicated sweet flavors were best used for positive emotional expression and coregulation [10,12]. Flavor-based memory cues were described in terms of ingredients $(75 \%, \mathrm{n}=18,10 \mathrm{FM}, 8 \mathrm{NFM})$, recipes (58\%, $\mathrm{n}=14,7 \mathrm{FM}, 7 \mathrm{NFM})$ and dishes (58\%, 8 FM, 6 NFM) often through combinations of, rather than single, ingredients. Most dishes consisted of known or existing combinations or recipes (11/14). However, in 3 cases these mentioned new, creative combinations of foods that commonly occur side-by-side but do not belong to a single dish such as coffee and flapjack: "you've got this bitter strong flavor and then you've got the sort of chewy sweeter flavor but not too sweet" (P2) or bacon and coffee (P3), fruitcake and champagne (P3).

The foods chosen for cues were most often self-made $(33 \%, \mathrm{n}=8$, 4 FM, 4 NFM) such as stewed apples (P1) and tuna and cucumber sandwich (P3). This perhaps reflects the extra value invested in food that is made by oneself and resembles the IKEA effect [33] in comparison to foods ordered or purchased. Other foodstuff used to co-design the cues included ready-made foods (3/24 cues), food made by somebody in the past (3), and catered foods (3). Interestingly, no restaurant foods were reported for NFM, whilst 2 were for FM, likely because they involve experiences in food centric settings whereas NFM were not related to a specific meal. Flavor-based cues were also described by the cooking style (11 times), which was used to specify the details of both self-made foods and those made by somebody else: "bacon, [my mother] used to cook it until it was an absolute crisp. She'd just leave it in the oven and it was very crispy. Still tasty, but different texture to how you have ever eaten bacon before" (P1). This allowed participants to elevate mundane foods towards specific flavor experiences, going beyond bacon as a ubiquitous experience to mum's very crispy bacon which has a specific and meaningful resonance, with particular odor and texture qualities.

For NFM, participants associated foods or flavors with their specific memories. Most associations were to foods that were temporally proximal to the memory being described (7/12), either during, before, or after the event of that memory. 3 memories were associated with food present during the event but not core to the memory, such as salted crisps on a trip to the seaside. Only one example related to food prepared before the memory. 3 memories were linked to food experienced after the event captured by these memories. After the event was not only the next thing eaten immediately after the memory ( 1 instance) but also longer term such as in the months or year following (2), often relating to foods eaten as a result of the impacts of the remembered event, i.e., food eaten as a result of having broken an arm (P4), or moving to University (P4).

Non-food memories were also associated with feelings generated by the event (3) such as joy and elation, including for instance two uses of champagne. Also present were associations to the favorite food of other living creatures (humans and a cat). Finally, one single food cue related to the place of the memory as something regularly consumed in that environment: ratatouille served in the university canteen (P4). 


\section{Discussion}

We now turn to the research questions set-out at the start of this paper. Firstly, what self-defining memories are chosen to be cued with a flavor-based cue? Our findings indicate that memories were mostly related to relationships (generally aligning with prior work $[28,34])$ and overwhelmingly positive. FM and NFM were mostly similar in nature, aside from a few emotionally negative NFMs. Our findings agree with prior work that flavor-based cues better supported positive emotions than negative ones [10,12], and indicate that flavor-based memory cues are more likely to be successful when prompting memorable positive experiences or events. The elicited self-defining memories were mostly socially shared, one-off events relating to experiences from adulthood. The prevalence of positive, social and relational memories with food indicates the character of food related memories to be wellsuited for enjoyable reminiscence that emphasize positive experiences and offer connection to social situations. This may be of particular use to older adults who may suffer from depression and social isolation [27]. Positive autobiographical memories also have the greatest potential for therapeutic use as indicated in studies on odor-evoked memories [12] and the proposed use of flavor as a memory cue could leverage this potential. One way that flavor-based memory cues may extend existing approaches is their potential value to cue events taking place not just in the reminiscence bump period, but throughout the entire lifespan.

The second question was; what flavor-based cues are designed for self-defining memories which include food and do not include food? In regard to all memories, umami, salty, and sweet taste-based cues were most popular, reporting a more nuanced relationship between positive experience and tastes than had been found in studies for more abstract emotion experiences in HCI [10,12]. The episodic and unique character of the memories appears to support more complex meaning-making with taste as part of flavor experiences that also comprise odors and textures. These taste findings align closely to the perspective on taste-emotion mappings of chefs and food designers [8]. Participants mostly opted to describe flavor-based cues through dishes, usually made by the person themselves, representing a personal engagement with food that extended beyond it being eaten. In previous work on flavor-based cues for emotional experience, both generic and idiosyncratic flavors were selected [12]. In this study, all the foods were complex and specific (and not generic) in part due to their relation to episodic moments. This specificity sometimes resulted in a creative construction of single flavor-cues which combined two normally separate but related foodstuffs such as coffee and flapjack. This combination of two common, yet independent flavors could be leveraged in future to create specific meaningful links between a memory and its cue. Specificity was also delivered through the cooking style which infused saliency to mundane foods, for instance, through the crispy bacon example we can see how the longer cooking times resulted in a different mix of odor compounds within the flavor experience created through extended heating [24].

In relation to NFM, the primary strategy employed to connect memories to food was temporal proximity. This indicates that specific memories are most readily related to specific foods that were present at, or around the time when the memorable event occurred. The foods from around a memory appear to be connected to the felt experience at the time. This is particularly apparent in the case of foods that were associated to short term diet changes following breaking an arm, or while undergoing cancer treatment (P4). Temporal proximity was not only used in isolation, as participants meaning making could be compound as well. For example, champagne's proximity to moments of celebration also creates an associated feeling that can be used to strengthen the connection between a memory and a flavor-based cue. Use of a person's preferences reflected previous work on designing flavors for user experience [12]. To conclude, our preliminary findings indicate that a range of co-design strategies were observed that can be drawn upon to refine the approach of future work on flavor-memory cues.

\section{Future Work}

This work presents an initial exploration into the co-design of flavor-based memory cues for self-defining memories in old age. Following the preliminary work presented here, a study is planned to produce and evaluate flavor-based cues with a larger sample and involve the production of flavor cues via 3D food printing. Through connecting edible experiences with digital technology it is also possible to design systems in which cueing can occur spontaneously [19] or be used as part of planned therapeutic programs [12]. Despite the suitability of odor cues for older adults, there remains questions to this group's acceptance of 3D printed food [9] therefore consideration of adoption will be important to increase impact.

\section{ACKNOWLEDGMENTS}

This work was supported by the Designing Multisensory Interactions: Emotional User Experience of 3D Printed-Food Consumption project (Grant Number 1962364) funded by EPSRC and Dovetailed Ltd. under a CASE PhD award.

\section{REFERENCES}

[1] Ferran Altarriba Bertran, Samvid Jhaveri, Rosa Lutz, Katherine Isbister, and Danielle Wilde. 2018. Visualising the Landscape of Human-Food Interaction Research. In Proceedings of the 2018 ACM Conference Companion Publication on Designing Interactive Systems (DIS '18 Companion), 243-248. https://doi.org/10.1145/3197391.3205443

[2] Artin Arshamian, Emilia Iannilli, Johannes C. Gerber, Johan Willander, Jonas Persson, Han-Seok Seo, Thomas Hummel, and Maria Larsson. 2013. The functional neuroanatomy of odor evoked autobiographical memories cued by odors and words. Neuropsychologia 51, 1: 123-131. https://doi.org/10.1016/j.neuropsychologia.2012.10.023

[3] Stephen Brewster, David McGookin, and Christopher Miller. 2006. Olfoto: Designing a Smell-based Interaction. In Proceedings of the SIGCHI Conference on Human Factors in Computing Systems (CHI '06), 653-662. https://doi.org/10.1145/1124772.1124869

[4] Jaz Hee-jeong Choi, Marcus Foth, and Greg Hearn (eds.). 2014. Eat, Cook, Grow: Mixing Human-Computer Interactions with Human-Food Interactions. The MIT Press, Cambridge, Mass. Retrieved November 2, 2016 from http://lib.myilibrary.com?id=586295

[5] Simon Chu and John J. Downes. 2004. Proust Reinterpreted: Can Proust's Account of Odour-cued Autobiographical Memory Recall Really be Investigated? A Reply to Jellinek. Chemical Senses 29, 5: 459-461. https://doi.org/10.1093/chemse/bjh056 
[6] E. L. Doets and S. Kremer. 2016. The silver sensory experience - A review of senior consumers' food perception, liking and intake. Food Quality and Preference 48, Part B: 316-332. https://doi.org/10.1016/j.foodqual.2015.08.010

[7] Tom Gayler. 2020. Inbodied interaction design example: smell. Interactions 27 , 2: 38-39. https://doi.org/10.1145/3380870

[8] Tom Gayler and Corina Sas. 2017. An Exploration of Taste-emotion Mappings from the Perspective of Food Design Practitioners. In Proceedings of the $2 \mathrm{Nd}$ ACM SIGCHI International Workshop on Multisensory Approaches to Human-Food Interaction (MHFI 2017), 23-28. https://doi.org/10.1145/3141788.3141793

[9] Tom Gayler, Corina Sas, and Vaiva Kalnikaitē. 2018. User Perceptions of 3D Food Printing Technologies. In Extended Abstracts of the $2018 \mathrm{CHI}$ Conference on Human Factors in Computing Systems (CHI EA '18), LBW621:1-LBW621:6. https://doi.org/10.1145/3170427.3188529

[10] Tom Gayler, Corina Sas, and Vaiva Kalnikaite. 2019. Taste Your Emotions: An Exploration of the Relationship Between Taste and Emotional Experience for HCI. In Proceedings of the 2019 on Designing Interactive Systems Conference (DIS '19), 1279-1291. https://doi.org/10.1145/3322276.3322336

[11] Tom Gayler, Corina Sas, and Vaiva Kalnikaite. 2020. Framing the Design Space for Food-Human-Technology Interaction. Forthcoming Publication.

[12] Tom Gayler, Corina Sas, and Vaiva Kalnikaite. 2020. Material Food Probe: Personalized 3D Printed Flavors for Emotional Communication in Intimate Relationships. In Proceedings of the 2020 ACM on Designing Interactive
Systems
Conference
(DIS
'20),
965-978. https://doi.org/10.1145/3357236.3395533

[13] Doménique van Gennip, Elise van den Hoven, and Panos Markopoulos. 2015 Things That Make Us Reminisce: Everyday Memory Cues As Opportunitie for Interaction Design. In Proceedings of the 33rd Annual ACM Conference on Human Factors in Computing Systems (CHI '15), 3443-3452. https://doi.org/10.1145/2702123.2702460

[14] Daniel L. Greenberg, Jennifer M. Ogar, Indre V. Viskontas, Maria Luisa Gorno Tempini, Bruce Miller, and Barbara J. Knowlton. 2011. Multimodal Cuing of Autobiographical Memory in Semantic Dementia. Neuropsychology 25, 1: 98 104. https://doi.org/10.1037/a0021005

[15] Aimee V. Hamel, Tai L. Sims, Dan Klassen, Thomas Havey, and Joseph E. Gaugler. 2016. Memory Matters: A Mixed-Methods Feasibility Study of a Mobile Aid to Stimulate Reminiscence in Individuals With Memory Loss Journal of Gerontological Nursing 42, 7: 15-24. https://doi.org/10.3928/00989134-20160201-04

[16] Rachel S. Herz. 1998. Are Odors the Best Cues to Memory? A Cross-Modal Comparison of Associative Memory Stimulia. Annals of the New York Academy of Sciences 855, 1: 670-674. https://doi.org/10.1111/j.17496632.1998.tb10643.x

[17] Rachel S. Herz. 2004. A Naturalistic Analysis of Autobiographical Memorie Triggered by Olfactory Visual and Auditory Stimuli. Chemical Senses 29, 3 217-224. https://doi.org/10.1093/chemse/bjh025

[18] Rachel S Herz, James Eliassen, Sophia Beland, and Timothy Souza. 2004 Neuroimaging evidence for the emotional potency of odor-evoked memory. Neuropsychologia $42, \quad 3$ : https://doi.org/10.1016/j.neuropsychologia.2003.08.009

19] Elise Hoven and Berry Eggen. 2008. Informing Augmented Memory System Design Through Autobiographical Memory Theory. Personal Ubiquitous Comput. 12, 6: 433-443. https://doi.org/10.1007/s00779-007-0177-9

20] Elise van den Hoven and Berry Eggen. 20140414. The cue is key: Design for real-life remembering. Zeitschrift für Psychologie 222, 2: 110. https://doi.org/10.1027/2151-2604/a000172

[21] Stephan Huber, Renate Berner, Martina Uhlig, Peter Klein, and Jörn Hurtienne. 2019. Tangible Objects for Reminiscing in Dementia Care. In Proceedings of the Thirteenth International Conference on Tangible, Embedded, and Embodied Interaction (TEI '19), 15-24. https://doi.org/10.1145/3294109.3295632
[22] Maria Larsson, Johan Willander, Kristina Karlsson, and Artin Arshamian. 2014 Olfactory LOVER: behavioral and neural correlates of autobiographical odor memory. Frontiers in Psychology 5. https://doi.org/10.3389/fpsyg.2014.00312

[23] Huy Viet Le, Sarah Clinch, Corina Sas, Tilman Dingler, Niels Henze, and Nigel Davies. 2016. Impact of Video Summary Viewing on Episodic Memory Recall: Design Guidelines for Video Summarizations. In Proceedings of the $2016 \mathrm{CH}$ Conference on Human Factors in Computing Systems - CHI '16, 4793-4805. https://doi.org/10.1145/2858036.2858413

[24] Donald S. Mottram. 1993. Flavor Compounds Formed during the Maillard Reaction. In Thermally Generated Flavors. American Chemical Society, 104126. https://doi.org/10.1021/bk-1994-0543.ch010

[25] Marianna Obrist, Alexandre N. Tuch, and Kasper Hornbaek. 2014. Opportunities for odor: experiences with smell and implications for technology. In Proceedings of the SIGCHI Conference on Human Factors in $\begin{array}{llll}\text { Computing } & \text { Systems } & \text { (CHI } & \text { '14), }\end{array}$ https://doi.org/10.1145/2556288.2557008

[26] S. Tejaswi Peesapati, Victoria Schwanda, Johnathon Schultz, Matt Lepage, Soyae Jeong, and Dan Cosley. 2010. Pensieve: Supporting Everyday Reminiscence. In Proceedings of the SIGCHI Conference on Human Factors in $\begin{array}{llll}\text { Computing } & \text { Systems } & \text { (CHI 10), 2027-2036. }\end{array}$ https://doi.org/10.1145/1753326.1753635

[27] Chengcheng Qu, Corina Sas, and Gavin Doherty. 2018. Exploring and Designing for Memory Impairments in Depression.

[28] Corina Sas. 2018. Exploring Self-Defining Memories in Old Age and Their Digital Cues. In Proceedings of the 2018 Designing Interactive Systems Conference (DIS '18), 149-161. https://doi.org/10.1145/3196709.3196767

[29] Corina Sas, Scott Challioner, Christopher Clarke, Ross Wilson, Alina Coman, Sarah Clinch, Mike Harding, and Nigel Davies. 2015. Self-Defining Memory Cues: Creative Expression and Emotional Meaning. In Proceedings of the 33rd Annual ACM Conference Extended Abstracts on Human Factors in Computing Systems (CHI EA '15), 2013-2018. ttps://doi.org/10.1145/2702613.2732842

[30] Corina Sas and Alina Coman. 2016. Designing personal grief rituals: An analysis of symbolic objects and actions. Death studies 40, 9: 558-569.

[31] Corina Sas, Nigel Davies, Sarah Clinch, Peter Shaw, Mateusz Mikusz, Madeleine Steeds, and Lukas Nohrer. 2020. Supporting stimulation needs in dementia care through wall-sized displays. In Proceedings of the $2020 \mathrm{CHI}$ Conference on Human Factors in Computing Systems, 1-16.

[32] Corina Sas, Tomasz Fratczak, Matthew Rees, Hans Gellersen, Vaiva Kalnikaite, Alina Coman, and Kristina Höök. 2013. AffectCam: Arousal- Augmented Sensecam for Richer Recall of Episodic Memories. In CHI '13 Extended Abstracts on Human Factors in Computing Systems (CHI EA '13), 1041-1046. https://doi.org/10.1145/2468356.2468542

[33] Corina Sas and Carman Neustaedter. 2017. Exploring DIY Practices of Complex Home Technologies. ACM Trans. Comput.-Hum. Interact. 24, 2: 16:1-16:29. https://doi.org/10.1145/3057863

[34] Jefferson Singer, Blerim Rexhaj, and Jenna Baddeley. 2007. Older, wiser, and happier? Comparing older adults' and college students' self-defining memories. Memory 15, 8: 886-898. https://doi.org/10.1080/09658210701754351

[35] Charles Spence. 2010. The multisensory perception of flavour. Psychologist 23 9: $720-723$.

[36] Elizabeth Thiry and Mary Beth Rosson. 2012. Unearthing the Family Gems: Design Requirements for a Digital Reminiscing System for Older Adults. In CHI '12 Extended Abstracts on Human Factors in Computing Systems (CHI EA '12), 1715-1720. https://doi.org/10.1145/2212776.2223698

[37] Johan Willander and Maria Larsson. 2006. Smell your way back to childhood Autobiographical odor memory. Psychonomic Bulletin \& Review 13, 2: 240 244. https://doi.org/10.3758/BF03193837

[38] Gesualdo M. Zucco, Lara Aiello, Laura Turuani, and Egon Köster. 2012. Odorevoked autobiographical memories: age and gender differences along the life span. Chemical Senses 37, 2: 179-189. https://doi.org/10.1093/chemse/bjr089 